\title{
Management and cause analysis of platelet transfusion refractoriness
}

\author{
Xiangyan Huang* \\ Department of Blood Transfusion, The 960th Hospital of PLA, Jinan 250031, Shandong China.
}

\begin{abstract}
Platelet transfusion refractoriness (PTR) can be defined as the less increment of platelet count than expected after platelets transfusion, which is a challenging and expensive problem often observed in platelet-transfusiondependent patients. Although PTR occurs most frequently due to non-immune causes, a significant minority is still caused by immune factors. The most important factor in immune dependent PTR is alloimmunization against Class I human leukocyte antigens (HLAs) or human platelet antigens (HPAs). The compatible platelets can be provided to immune-mediated patients using platelet crossmatching, HLA matching, and antibody specificity testing. These measures-aimed to eliminate donor-specific HLA antibodies will lead to the improved clinical management of PTR patients, caused by severe alloimmunization.
\end{abstract}

Keywords: platelet transfusion refractoriness; alloimmunization; management

Platelets play an indispensable role in maintaining hemostasis, so platelet transfusion is now commonly used to boost the number of platelets in patients with both qualitative and quantitative platelet defects. Platelet transfusion refractoriness (PTR) represents a decreased platelet count following transfusion, which is significantly less than expected. This review will address the definition of PTR, causes of PTR and strategies for managing PTR patients.

\section{DEFINITION OF PLATELET TRANSFU- SION REFRACTORINESS}

PTR can be simply defined as the lower increase in platelet count than expected following platelets transfusion. The more precise definition of PTR is the 1-hour posttransfusion corrected count increment (CCI) of less than 5000 to $7500 / \mu \mathrm{L} / \mathrm{m}^{2} / 10^{11}$ after two consecutive transfusions of $\mathrm{ABO}$-compatible platelets, at least one of which had been stored for

*Correspondence to: Xiangyan Huang, PhD, Director of Department of Blood Transfusion, The 960th Hospital of PLA, Jinan, 250031, PR China.E-Mail:xiangyan73@aliyun.com.Tel:+86-15969700616,Fax: +86-0531-51666968 no more than 48 hours $^{[1-2]}$. The criteria proposed in the $19^{\text {th }}$ edition of the $A A B B$ Technical Manual is a range rather than a numerical value, due to different studies adopting different limits based on the characteristics of the patients studied.

The most commonly used indicators for assessing the effectiveness of platelet transfusions are CCI and post-transfusion platelet recovery (PPR) measurements, and absolute post-transfusion platelet count increments (CI)- which is now accepted as more intuitive than $\mathrm{CCI}$ or $\mathrm{PPR}^{[3]}$. The calculation formulas are shown ${ }^{[4]}$ :

$\mathrm{CI}=$ posttransfusion platelet count - pretransfusion platelet count;

$$
\begin{aligned}
& \mathrm{CCI}=\frac{\mathrm{CI}(/ \mu \mathrm{L}) \times \text { body surface } \operatorname{area}\left(\mathrm{m}^{2}\right)}{\text { platelets transfused }\left(10^{11}\right)} \\
& \mathrm{PPR}=\frac{\mathrm{CI}(/ \mathrm{mL}) \times \text { patient blood volume }(\mathrm{mL})}{\text { platelets transfused }} \times 100 \%
\end{aligned}
$$

The CCI formula considers both the number of platelets transfused and the body surface area of the patient. Although most experts would agree with the precise definition of PTR as described above, platelet transfu- 
sion can generally be diagnosed as effective if 1-hour CCI $\geqslant 7500$ and 18- to 24-hour CCI $\geqslant 4500$. The timing of post transfusion platelet count is crucial for the interpretation of CCI, since the time can indicate the reason for $\mathrm{PTR}^{[2]}$. A low 1-hour CCI after transfusion usually indicates that the PTR is caused by antibody-mediated platelet destruction, severe splenic sequestration and/or massive bleeding ${ }^{[1]}$. It is also possible that PTR is due to a combination of nonimmune factors. A reduced CCI at 18 to 24 hours with a normal CCI at 1 hour suggests that other nonimmune clinical factors have led to increased platelet consumption $^{[2,5]}$. A 10-minute post-transfusion CCI can be used to determine PTR following the criteria of 1-hour $\mathrm{CCI}^{[2]}$.

The PPR formula takes into account both the number of platelets transfused and blood volume. It is currently recommended that the following criteria is used to insure successful platelet transfusion: 1-hour post-transfusion PPR of more than $30 \%$ and 20-to 24hour post-transfusion PPR of more than $20 \%{ }^{[6]}$.

\section{THE FACTORS LEADING TO PTR}

About $20 \%$ to $70 \%$ of multi-transfused patients with thrombocytopenia show a less-than-expected increment in platelet count ${ }^{[1]}$. The platelet transfusion for hematological malignancies is particularly likely to result in $\mathrm{PTR}^{[1]}$. There are many factors that lead to PTR, which are divided into two broad categories:immune factors and non-immune factors ${ }^{[7]}$. Although immune factors are often encountered as causes of refractoriness, non-immune causes are more likely to affect a poor response to platelet transfusion ${ }^{[1-2,8-9]}$. In addition, immune causes of refractoriness are often simultaneously present with non-immune factors ${ }^{[10]}$.

\section{Non-immune causes}

According to a report by Forest et $a l^{[2]}$, about twothirds of platelet refractoriness have non-immune causes, while another $20 \%$ are caused by a combination of immune and non-immune factors. These non-immune causes include many factors, such as platelet storage quality, sepsis, disseminated intravascular coagulation, and thrombotic thrombocytopenic purpura. The most commonly cited non-immune factors ${ }^{[1,2,11]}$ leading to refractoriness are listed: sepsis,infection,fever,massive bleeding,splenomegaly (splenic sequestration), hypersplenism, disseminated intravascular coagulation,medications (may include immune mechanisms), intravenous amphotericin b, poor storage of platelets, allogeneic transplantation and treatment regimen (ie, total body irradiation or chemotherapy),graft-versus-host disease, and thrombotic thrombocytopenic purpura.

Careful clinical observation and active treatment of potential diseases are ways to relieve non-immune factors for platelet refractoriness. In addition, platelet product characteristics should be paid more attention to, such as dose, the method of collection (apheresis or pooled), donor-recipient ABO compatibility and platelet storage period, which may also affect platelet count increment after transfusion ${ }^{[12]}$.

Although non-immune causes are more likely to cause PTR, little can be done except for recommending the treatment of the disorder. Therefore, the review focuses on immune-related causes of platelet refractoriness.

\section{Immune causes}

Although Immune factors lead to a minority (20\% 25\%) in PTR, immune factors are still a significant proportion and discussed more in the field of blood transfusion ${ }^{[11,13]}$. Immune-mediated factors include platelet alloantibodies [including anti-human leucocyte antigen (HLA) antibody, anti-human platelet antigen (HPA) antibody, anti-ABO antibody, anti-platelet glycoprotein antibody in patients with congenital deficiency], platelet autoantibodies and drug-dependent platelet antibodies ${ }^{[7]}$.

\section{Platelet alloantibodies}

Platelets express many kinds of antigenic markers on their surface. Some of these antigensare shared with other cells, like ABO and Class IHLA, while other antigens are essentially platelet-specific, such as $\mathrm{HPA}^{[1]}$. The most important immune-mediated factors include antibodies to ABO, Class I HLA (mostly HLA-A and HLA-B), and HPA ${ }^{[14]}$. Antibodies to CD36 in CD36-deficient individuals exposed to normal platelets also have been reported to cause $\mathrm{PTR}^{[15,16]}$.

Blood group A and B antigens are expressed on platelets, and antigen levels are quite variable in different individuals, with $5 \%$ to $10 \%$ of non-group$\mathrm{O}$ individuals expressing extremely strong $\mathrm{Al}$ or $\mathrm{B}$ antigen on their platelets ${ }^{[17-19]}$. Antibodies to $\mathrm{ABH}$ antigens can adversely affect platelet transfusion recovery and survival in ABO-incompatible platelet transfusion. It is worth noting that the donors with the subgroup A2 red cell phenotype possess a Bombay-like phenotype with lacking both $\mathrm{A}$ and $\mathrm{H}$ antigens and do not express detectable $\mathrm{A}$ antigens on their platelets ${ }^{[18,20]}$. As a result, Platelets from group A2 may be a superior product as a selection of 
crossmatched or HLA-matched platelets for group O patients, even those with high-titer anti-A.

The prevalence of HLA/HPA polymorphisms in various populations showed significant differences. Prior exposure to transfusion, pregnancy or transplantation can lead to alloimmunization to HLA and/ or HPA. Immune platelet refractoriness is mostly due to HLA antibodies, rather than HPA antibodies ${ }^{[21-22]}$.

The predominant Class I HLA antigens expressed on platelets are HLA-A and HLA-B locus antigens, while HLA-C antigens appear to be only present on minimal platelets ${ }^{[23]}$. However, antibodies to HLA$\mathrm{C}$ antigens can still be a cause of PTR ${ }^{[24-25]}$. Class II HLA is not expressed on the platelet membrane. HLA alloimmune platelet refractoriness can lead to serious consequences ${ }^{[26]}$. Pregnancy increases the risk of HLA alloantibody formation, and the frequency of HLA alloimmunization in women who have had at least two pregnancies is often more than $20 \%{ }^{[27]}$. For patients with repeated blood transfusions, leukocyte in blood productsis an important factor in the development of HLA alloimmunization. HLA antibodies can be found in 25\% 30\% of transfused leukemic patients and can be detected in as high as $80 \%$ of aplastic anemia patients ${ }^{[28]}$. The use of leuko-reduced blood products have been adopted in most hospitals and blood centers, which reduce almost 50\% HLA alloimmunization $^{[29]}$.

HPAs are platelet specific alloantigens. Currently, 35 different HPAs have been identified on six different platelet membrane glycoproteins-GPIIb, GPIIIa, GPIb $\alpha$, GPIb $\beta$, GPIa, and CD $109^{[1,30]}$. The incidence of platelet-specific antibodies varies from $2 \%$ to $11 \%$, which cannot be affected by leukoreduction ${ }^{[2]}$. HPA antibodies can cause refractoriness to platelet transfusion, but most of them are usually confounded by concurrent HLA antibodies ${ }^{[2,31]}$.

\section{Platelet autoantibodies}

In addition to causing immune thrombocytopenic purpura, autoantibodies can also cause transfusion refractoriness, such as PTR related to an autoantibody to platelet glycoprotein ${ }^{[7]}$. In this condition autoantibodies are directed against platelet antigens from both autologous platelets and transfused platelets, resulting in platelet destruction.

\section{Drug-inducedplatelet antibodies}

PTR can be caused by drug-induced platelet an tibodies-a recognized complication of drug therapy. Although there are more than 100 drugs reported to be associated with causing this, drugs most frequently implicated include quinine, quinidine, sulfa drugs, vancomycin, GP $I \mathrm{~b} / \mathrm{II}$ a antagonists, heparin, carbamazepine, eptifibatide, ibuprofen, oxaliplatin, rifampin, trimethoprim and vancomycin ${ }^{[1,32-33]}$. These drugs may produce both drug-dependent and non-drug-dependent antibodies. Among them, drugdependent antibodies are able to recognize the conformational changes induced by the drug, which interacts with platelet membrane glycoproteins ${ }^{[34-35]}$. These antibodies can cause a sudden and rapid onset of thrombocytopenia, which is usually resolved within 3 to 4 days after drug withdrawal.

Briefly, a lack of response to platelet transfusion can be caused by a combination of factors related to the patient and to platelet concentrate. This makes it often difficult to separate immune and non-immune factors because patients receiving platelets often have multiple patient-related factors ${ }^{[8,36]}$.

\section{STRATEGIES FOR MANAGING PTR PATIENTS}

\section{General strategies}

Potential non-immune factors should be paid close attention. Any correctable clinical factors which may cause platelet refractoriness should be treated. The correct dose of platelets should be given to the patient. ABO identical and freshest (at least one transfusion of platelets within 48 hours) available platelets should be used when possible. The patient's response to the platelets should be carefully observed to see whether the platelet count is improved $^{[2,28]}$.

If the above steps have failed and a poor response to platelet transfusion is found, crossmatchcompatible or HLA-matched platelets (whichever is available soonest) should be given. Any evidence for immune related platelet refractoriness should be assessed by detecting the presence of HLA or HPA antibodies or both, which also may be of value inmaking future decisions in the PTR patient. If HLA or HPA antibodies are identified, crossmatching, HLA matching, and antibody specificity prediction should be used for identifying compatible platelet units $^{[2,11,28]}$. Strategies for managing PTR patients are listed in Figure 1.

\section{Strategies for PTR caused by alloimmunization}

\section{Platelet cross matching}

Platelet crossmatching (useful for HLA and/or HPA antibodies) is performed by incubating donor platelets with recipient plasma and testing for crossmatch compatibility ${ }^{[23]}$. Crossmatching supports the 


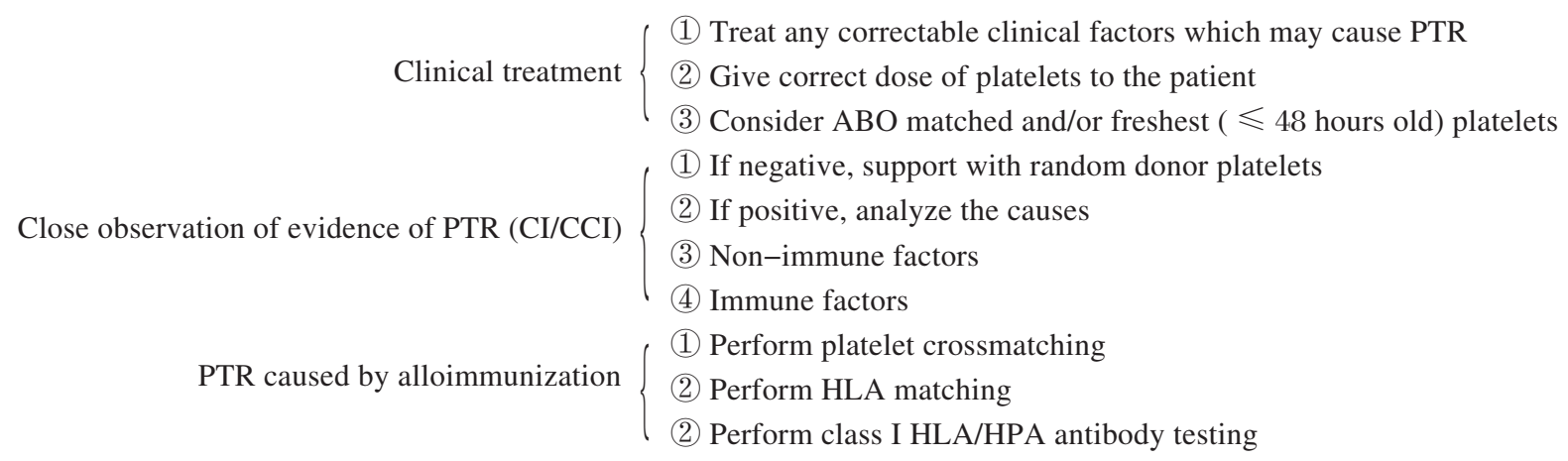

Figure 1 Strategies for managing PTR patients

rapid and effective selection of donor platelets compared with HLA matching ${ }^{[22]}$. This method is often used because of its rapidity and feasibility, but the shortcomings of this method are also worth noting. Finding matched platelets in highly sensitized patients is often difficult using platelet cross matching, which can cause a continued risk of alloimmunization if the HLA platelet is mismatched.

\section{HLA matching}

Most PTR is caused by immune factors in patients immunized to HLA, since immunization to HPA is much less frequent ${ }^{[28]}$. In highly sensitized patients, the first option is to use HLA-matched platelets ${ }^{[2,37]}$. HLA matching is performed by HLA typing of the patient and providing platelets collected from an HLA matched donor, which includes different degree based on a classification system described in Table 1. A higher degree of match (for example, grade A, $\mathrm{B} 1 \mathrm{U}$, and B2U matches) leads to better count increments, and is therefore better at preventing future alloimmunization ${ }^{[37-38]}$. However, this method is also limited because of a requirement for a large donor pool which is not easy to achieve ${ }^{[2]}$.

Table 1 HLA-match grade classification

\begin{tabular}{ll}
\hline Grade & \multicolumn{1}{c}{ Degree of matching } \\
\hline A & All 4 donor HLA loci are identical to the recipient \\
B1U & Only 3 antigens are detected in donor and all 3 detected antigens are identical to the recipient \\
B1X & 2 HLA antigens are identical to the recipient and 1 HLA antigen is incompatibility \\
B2U & Only 2 antigens are detected in the donor and both detected antigens are identical to the recipient \\
B2UX & Only 3 HLA antigens are detected in the donor, among them, 2 antigens are identical withthe recipient and 1 antigen is incompatibility \\
B2X & 2 antigens are identical to the recipient and 2 antigens are incompatibility \\
$\mathrm{C}$ & 3 antigens identical to recipient and one non cross-reactive antigen of donor is not present in the recipient \\
$\mathrm{D}$ & 2 antigens of the donor are not present in the recipient and show non cross-reactive mismatches \\
$\mathrm{R}$ & Random donor
\end{tabular}

\section{HLA/HPA antibody testing}

Class I HLA/HPA antibody testing could provide another option in selecting HLA/HPA antigen-restricted platelets, that avoid the HLA/HPA antibodies $^{[11,37]}$. This method provides platelets without specific HLAs/HPAs to the detected antibodies, which has the advantage of creating a larger donor pool and increased feasibility, since only the patient's HLA/ HPA antibodies need to be known rather than the HLA typing. This approach expands the range of finding compatible platelets, but also has potential risk of alloimmunization against mismatched donor HLAs. Another inconvenience is that donor HLA/ HPA should be typed. HLA and/or HPA antibodies can be detected by using various techniques including flow cytometry (FCM), Luminex, modified antigen capture ELISA (MACE), monoclonal antibody specific immobilization of platelet antigens assay (MAIPA), simplified sensitized e-rythrocyte platelet serology assay (SEPSA), and solid phase red cell adherence assay (SPRCA) ${ }^{[28,39-41]}$.

\section{Strategies for PTR caused by severe alloim- munization}

PTR is a complex complication in platelet-transfusion-dependent patients, especially in the patients with severe HLA alloimmunization which makes it extremely difficult to find compatible platelets ${ }^{[42-43]}$. Notably, most HLA antibodies can be found against 
public epitopes, which are shared by two or more antigens ${ }^{[37,44]}$. This is why the patients with HLA antibodies cross-react with numerous HLA alleles.

PTR caused by severe alloimmunization remains a therapeutic challenge in the management of patients who are dependent on platelet transfusion ${ }^{[45]}$. Although HLA-matched platelets are a priority choice for most HLA alloimmunized patients, $20 \% \sim 50 \%$ of patients may remain refractoriness-even with matched platelets $^{[42]}$. For these patients with PTR caused by severe alloimmunization, the platelet transfusion management method needs to be changed. That is, instead of ensuring matching platelets, the elimination of donor-specific HLA antibodies should be sought.

Alternative management strategies have been reported to overcome the complication. Recent studies ${ }^{[2,42,46]}$ have outlined treatments for PTR patients who are highly sensitized to HLA antigens, such as intravenous immunoglobulins (IVIG), plasma exchange (PE), protein A column therapy, rituximab, or IgG endopeptidase (IdeS). These alternative strategies have been reported mainly in case reports, and may produce different effects in different individuals. They may yet lead the future of PTR treatment, however for now more unambiguous recommendation and wider experimental evidence are required.

\section{Acknowledgements and funding}

This work is supported by the grant from the General Hospital of Jinan Military Command Foundation (No.2016ZD05).

\section{References}

[1] Fung MK. Technical Manual[M]. 19th ed. Bethesda, Maryland: AABB, 2017.

[2] Forest SK, Hod EA. Management of the Platelet refractory patient[J]. Hematol Oncol Clin N Am, 2016,30(3):665-677

[3] Slichter SJ, Davis K, Enright H, et al. Factors affecting posttransfusion platelet increments, platelet refractoriness, and platelet transfusion intervals in thrombocytopenic patients[J]. Blood, 2005,105(10):4106-4114

[4] Fung MK. Technical Manual[M]. 18h ed. Bethesda, Maryland: $A A B B, 2014$.

[5] Delaflor-Weiss E, Mintz PD. The evaluation and management of platelet refractorinessand alloimmunization[J]. Transfus Med Rev,2000,14(2):180-96

[6] Rebulla P. Formulae for the definition of refractoriness to platelet transfusion[J]. Transfus Med,1993,3(1):91-93

[7] Stanworth SJ, Navarrete C, Estcourt L, et al.Platelet refractoriness--practical approaches and ongoing dilemmas in patient management $[\mathrm{J}]$. Br J Haematol,2015,171(3):297-305

[8] Hod E, Schwartz J. Platelet transfusion refractoriness[J].
Br J Haematol,2008,142(3):348-360

[9] Vassallo RJ. New paradigms in the management of alloimmune refractoriness to platelet transfusions[J]. Curr Opin Hematol,2007,14(6):655-663

[10] Tanoue S, Konuma T, Kato S, et al. Platelet transfusion refractoriness in Single-Unit cord blood transplantation for adults: risk factors and clinical outcomes[J]. Biology of Blood and Marrow Transplantation,2018,24(9):1873-1880

[11] Juskewitch JE, Norgan AP, De GS, et al. How do I[J]. manage the platelet transfusion-refractory patient?[J]. Transfusion,2017,57(12):2828-2835

[12] Triulzi DJ, Assmann SF, Strauss RG, et al. The impact of platelet transfusion characteristics on posttransfusion platelet increments and clinical bleeding in patients with hypoproliferative thrombocytopenia[J]. Blood,2012,119(23):5553-5562

[13] Doughty HA, Murphy MF, Metcalfe P, et al. Relative importance of immune and non-immune causes of platelet refractoriness[J]. Vox Sang,1994,66(3):200-205

[14] Abraham AS, Chacko MP, Fouzia NA, et al. Antibodies to human platelet antigens form a significant proportion of platelet antibodies detected in Indian patients with refractoriness to platelet transfusions[J]. Transfus Med,2018,28(5):392-397

[15] Xia W, Ye X, Xu X, et al. Two cases of platelet transfusion refractoriness and one case of possible FNAIT caused by antibodies against CD36 in China[J]. Transfus Med,2014,24(4):254-256

[16] Curtis BR, Ali S, Glazier AM, et al. Isoimmunizationagainst CD36(glycoprotein IV):Descriptionof four cases of neonatal isoimmunethrombocytopenia and brief review of the literature[J]. Transfusion,2002,42(9):1173-1179

[17] Curtis BR, Edwards JT, Hessner MJ, et al. Bloodgroup a and B antigens are strongly expressedon platelets of some individuals[J]. Blood,2000,96(4):1574-1581

[18] Cooling LL, Kelly K, Barton J, et al. Determinants of $\mathrm{ABH}$ expression on human bloodplatelets[J]. Blood,2005,105(8):3356-3364

[19] Josephson CD, Castillejo MI, Grima K, et al. ABOmismatched platelet transfusions: strategies to mitigate patient exposure to naturally occurring hemolytic antibodies[J]. Transfus Apher Sci,2010,42(1):83-88

[20] Skogen B, Rossebo HB, Husebekk A, et al. Minimal expression of bloodgroup A antigen on thrombocytes from A2 individuals[J]. Transfusion,1988,28(5):456-459

[21] Laundy GJ, Bradley BA, Rees BM, et al. Incidence and specificity of HLA antibodies in multitransfused patients with acquired aplastic anemia[J]. Transfusion,2004,44(6):814-825

[22] Sacher RA, Kickler TS, Schiffer CA, et al. Management of patientsrefractory to platelet transfusion[J]. Arch Pathol Lab Med,2003,127(4):409-414

[23] Kopko PM, Warner P, Kresie L, et al. Methods for the selection of plateletproducts for alloimmunerefractorypatients[J]. Transfusion,2015,55(2):235-244

[24] Saito S, Ota S, Seshimo H, et al. Platelet transfusionrefractoriness caused by a mismatch inHLA-C antigens[J]. 
Transfusion,2002,42(3):302-308

[25] Stanworth SJ, Hudson CL, Estcourt LJ, et al. TOPPS study investigators:Risk of bleeding and use of platelet transfusionsin patients with hematologic malignancies:recurrent eventanalysis[J]. Haematologica,2015,100(6):740-747

[26] Peña JR, Saidman SL, Girouard TC, et al. Anti-HLA alloantibodies in surgical patients refractory to platelet transfusion[J]. Am J Hematol,2014,89(9):E133-E137

[27] Triulzi DJ, Kleinman S, Kakaiya RM, et al. The effect of previous pregnancy and transfusion on HLA alloimmunization in blood donors: implications for a transfusion-related acute lung injury risk reduction strategy[J]. Transfusion,2009,49(9):1825-1835

[28] McCullough J. Transfusion Medicine[M]. 4th ed. Chichester, West Sussex; Hoboken, NJ: John Wiley \& Sons, Inc., 2017.

[29] Trial to Reduce Alloimmunization to Platelets Study Group. Leukocyte reduction and ultraviolet B irradiation of plateletsto prevent alloimmunization and refractoriness to platelettransfusions[J]. N Engl J Med, 1997, 337(26):1861-1869.

[30] Davey S, Navarrete C, Brown C. Simultaneous human platelet antigen genotyping and detection of novel single nucleotide polymorphisms by targeted next-generation sequencing[J]. Transfusion,2017,57(6):1497-1504

[31] Pappalardo PA, Secord AR, Quitevis P, et al. Platelet transfusion refractorinessassociated with HPAla(Pl(A1))alloantibody without coexistent HLA antibodiessuccessfully treated with antigen-negative platelet transfusions[J]. Transfusion,2001,41(8):984-987

[32] Reese JA, Li X, Hauben M, et al. Identifying drugs that cause acute thrombocytopenia: an analysis using 3 distinct methods[J]. Blood,2010,116(12):2127-2133

[33] Curtis BR. Drug-inducedimmune thrombocytopenia:inci dence,clinical features,laboratory testing, and pathogenic mechanisms[J]. Immunohematology,2014,30(2):55-65

[34] Aster RH, Bougie DW. Drug-induced immunethrombocy topenia[J]. N Engl J Med,2007,357(6):580-587

[35] Bougie DW, Wilker PR, Aster RH. Patients withquinine-induced immune thrombocytopeniahave both"drug-dependent"and"drug-specificantibodies[J].
Blood,2006,108(3):922-927

[36] Rioux-Massé B, Cohn C, Lindgren B, et al. Utilization of cross-matched or HLA-matched platelets for patients refractory to platelet transfusion[J].Transfusion,2014,54(12):3080-3087

[37] Schmidt AE, Refaai MA, Coppage M. HLA-mediated platelet refractoriness: an aclps critical review[J]. Am J Clin Pathol,2018. doi: 10.1093/ajcp/aqy121.

[38] Nambiar A, Duquesnoy RJ, Adams S, et al. HLAMatchma ker-drivenanalysis of responses to HLA-typed platelet transfusionsin alloimmunized thrombocytopenic patients[J]. Blood,2006,107(6):1680-1687

[39] Engelfriet CP, Reesink HW. Detection of platelet-eactive antibodies inpatients who are refractory to platelet transfusions, and the selection ofcompatible donors[J]. Vox Sang,2003,84(1):73-88

[40] Ma YT, Zq L, Wang QL. The progress of platelet antibody detection technology[J]. Chinese Journal of Laboratory Medicine,2006,29(11):1041-1044(in Chinese)

[41] Metcalfe P. Platelet antigens and antibody detection[J]. Vox Sang,2004,87(S1):582-586

[42] Cid J, Magnano L, Acosta M, et al. Rituximab, plasma exchange and intravenous immunoglobulins as a new treatment strategy for severe HLA alloimmune platelet refractoriness[J]. Platelets,2015,26(2):190-194

[43] Pena JR, Sudhof L, O’brien B. Peripartum management of HLA alloimmune platelet refractoriness[J]. Transfusion,2018,58(7):1583-1587

[44] EI-Awar N, Jucaud V, Nguyen A. HLA epitopes: the targetsof monoclonal and alloantibodies defined[J]. J Immunol Res, 2017, 2017:3406230.

[45] Solves P, Sanz J, Freiria C, et al. Factors influencing platelet transfusion refractoriness in patients undergoing allogeneic hematopoietic stem cell transplantation[J]. Ann Hematol,2018,97(1):161-167

[46] Burner JD, Goldfinger D. Potential use of IgG endopeptidase in the management of platelet refractoriness due to HLA alloimmunization[J]. Transfus Apher Sci,2018, 57(3):409-410.

(Received 11 November 2018, Revised 05 December 2018, Accepted 12 December 2018) 